\title{
A Hybrid WOFOST and Cropsyst Model for the Prediction of Crop Yield
}

\author{
Achir Jerome Aondongu \\ Department of Mathematics, \\ Statistics and Computer Science, \\ University of Agriculture Makurdi \\ Benue State, Nigeria.
}

\author{
Iorshase Agaji \\ Department of Mathematics, \\ Statistics and Computer Science, \\ University of Agriculture Makurdi \\ Benue State, Nigeria.
}

\author{
Esiefarienrhe Bukohwo M \\ Department of Mathematics, \\ Statistics and Computer Science, \\ University of Agriculture Makurdi \\ Benue State, Nigeria.
}

\begin{abstract}
Food availability is essential for any nation. The availability of food in turn depends on so many factors. In this work a hybrid model for the prediction of crop yield was proposed. This model combines the WOFOST and Cropsyst models and incorporated a crisis situation for prediction of crop yields. Factors used in the proposed model for prediction of crop yields include weather conditions, soil fertility, and crisis data. Crisis determines the availability of land for farming. Benue state of Nigeria was chosen as a study area. This choice is due to the fact that the state is tagged the food basket of Nigeria because of its mass production of many varieties of food crops. The crops covered in the study are maize, rice, tomato, millet, sorghum, beans. The new model was simulated using Visual Basic 2010 and results indicated that the model performed accurate predictions as compared to manual predictions. The system is recommended for use by food security agencies to avert hunger and starvation in future.
\end{abstract}

Keywords: WOFOST, CROPSYST, Hybrid, yield, crops, model, nutrient, computer

\section{INTRODUCTION}

Food security is a worldwide problem that has called the attention of all stakeholders in the agricultural sector. There is need to know the total crops yield in any economy. Such knowledge aids in planning in terms of agricultural inputs, storage facilities as well as processing.

Benue State is the Food Basket of the Nation and achieving food sufficiency in the state will go a long way to address most of food insecurity problems facing Nigeria.

According to Nwajiuba (2012), Nigeria has the ambition of diversifying her economy from crude petroleum dependency. The country also faces a looming food security crisis with a growing population that is increasingly dependent on imported foods. The once dominant subsistence-oriented farm economy is at risk of gradual marginalization. There are many models and methodologies used in the prediction of crop yields. In this work two such models are studied in details. These are World Food Studies (WOFOST) and Cropping System (Cropsyst). Direct application of the two models individually or in combination may not yield the desired result because of the prevailing crisis in Benue state. Therefore we combined WOFOST and Cropsyst and added a crisis component on the hybrid model and used it to predict crop yields for selected years in Benue State.

\section{LITERATURE REVIEW}

Crop yield predicting and forecasting enable stakeholders to take crucial decisions especially if such predictions and forecasts are carried out before harvest. Stakeholders in agriculture in Nigeria include the various governments (Federal, states and local), investors in the sector, farmers and researchers in the agricultural sector. The decisions may include planning for storage, processing and import-export plans. In this review we dwelt mostly on the 
International Journal of Computer Applications Technology and Research

Volume 8-Issue 01, 04-12, 2018, ISSN:-2319-8656

various models and methodologies used for predicting and forecast of yields.

Anup et al (2006) used pairwise linear regression method with breakpoint and utilized 19 years of data comprising normalized difference vegetation index(NDVI), rainfall soil moisture and surface temperature to assess and predict crop yield for Iowa state, USA. Their model did not, however, consider the crisis situation such as in Nigeria where insurgents and herdsmen clash with farmers over land utilization, land being a vital resources for farming.

Kefaya et al (2011) explored the dynamics of neural networks in forecasting crop yield using environmental factors like temperature, $\mathrm{Co}_{2}$, vapor pressure deficit and radiation. Their model has only yield as the output mode.

Dahikar and Rode (2014) Used artificial neural networks and parameters related to soil and atmosphere to predict crop yield. Their paper demonstrated the ability of ANN technology to be used to predict crop yields at rural district

Verma et al (2015) used a mixed model procedure as a way of fitting a multi-level model for crop yield prediction. Their paper also demonstrated the usefulness of their mixed model framework for preharvest crop yield forecasting.

Li et, al (2007) used ANN to predict corn and soya beans yields in the "corn belt" area of the Midwestern and Great Plains region of the United States. A new procedure that utilized SCE-UA algorithm was used to train the ANN. Their method had a prediction accuracy that was as high as 85 percent

Hoogenboom and Gresham (1993) analyzed crop models SOYGRO, PNUTGRO, CERES-maize and CERES-wheat and linked the models with a geographic information system. Their system was to simulate crop growth and development for each field and also predict yield and other crop input and output.

Conor and Mikahail (2005) used a stochastic weather generator to produce a probabilistic ensemble of water series which was used with a crop simulation model to generate distribution of crop characteristics. They analyzed six sites in Europe and Australia and demonstrated that there is a great variation in lead times for predicting selected crop characteristics between sites.

Galan et al (2008) sought to identify factors influencing olive fruit production in Andalusia Spain. They modeled pollen release and used field floral phenology data as well as meteorological data over the fruiting season. Their models provided earlier and more accurate crop estimates than those used by the Andalusian government.

Kushwaha and Sweta B (2015) tried to predict crop yield by processing Big Data using the Hadoop platform. Their work focused on soil type and the suitability of each crop for a given soil type. Their work identified good quality and improved crop yield using Agro algorithm implemented in Hadoop platform.

Wang etal (2012) developed a computer vision based system for crop yield estimation. Their system used a two-camera rid for the acquisition of images and a computer vision algorithm to generate apple count as crop yield estimation. Their results indicated that their system worked well with both red and green apples with minimum errors.

Savin etal (2007) examined the possibilities of using fuzzy neural network for predicting crop yield in Rostov oblast, Krasnodov and Stavropol krais regions of Russia. They used results from the simulation of CGMS model and values of vegetation index from SPOT VEGETATION satellite data as input to their model. Results from their work indicated 74 percent accuracy in predicting yield. Their result was however more suitable for sowing-flowering rather than sowing-ripening period.

Bornn and Zidek (2012) described how to incorporate spatial dependence into statistical models for crop yield. They created a Bayesian model which provided efficient predictions over least square methods. The also examined a range of possible dimension-reduction schemes as well as basic expansions to improve predictions. Their model also provided straightforward interpretation of the effects of climate on its output.

Sawasawa (2003) applied space- borne satellite based NDVI to predict rice yield at Andhra Pradesh state in India. His results indicated a significant correlation between remotely sensed NDVI and field level rice yield. His result revealed that land and management factors also significantly affect yield

Stockle (1996) developed CropSyst (Cropping Systems)

Model. The model considered crop water uptake and actual crop transpiration as equal. The inputs of the model include weather file, location, soil, crop and management data files. It predicted yield (tons) as a product of nitrogen-limited biomass and harvest index. The model is limited by the fact that it cannot simulate crop- 
specific potential evapotranspiration. It cannot also simulate crop water uptake different from actual crop transpiration. Subsequently the model neglected water retained by plant for turgidity and it placed no water stress in the estimation of crop evapotranspiration

Bouman et al. (1996) reported the development of World Food Studies (WOFOST) crop model. The model is site-specific and considered ecological factors under the assumption that optimum management practices were applied .It used maximum and minimum temperatures, rainfall, wind speed, water vapor pressure, global radiation and evapotranspiration as its inputs and predicted yield as a product of dry matter rate of the shoots and translocation factor of crop organ. The model is site and cultivar specific and that limits it general application.

The models and methodologies reviewed did not envisage the crisis situation which is now a major factor determining crop yield in Benue state, Nigeria. Apart from lack of available land for farming occasioned by crisis, crops are often destroyed by crisis. Therefore our aim in this paper is to build in crisis parameters in a hybridized WOFOST and Cropsyst model and to determine if it will improve crop yield prediction in crisis prone region having extremely fertile lands and climate for agriculture purposes.

\section{METHODOLOGY}

The WOFOST and Cropsyst models were studied in this work and their inadequacies observed.

The work proposed a new model to incorporate the strength of the two models, in addition, it incorporated the crisis situation which was not envisaged by both models.

The frequent occurrence of crisis within the Benue state has affected food production as hectares of land available for cultivation are usually reduced by crisis. A crisis sub-model was included in the hybrid model to estimate the number of hectares affected by crisis.

\subsection{Design of the Proposed System}

We began the design of the model with the design of its architecture as it gives a block structure of the system without specifying system details. The architecture of the new model is as shown is figure 1 . The model is a 3 -tier architecture made up of the front-end, logic and backend.

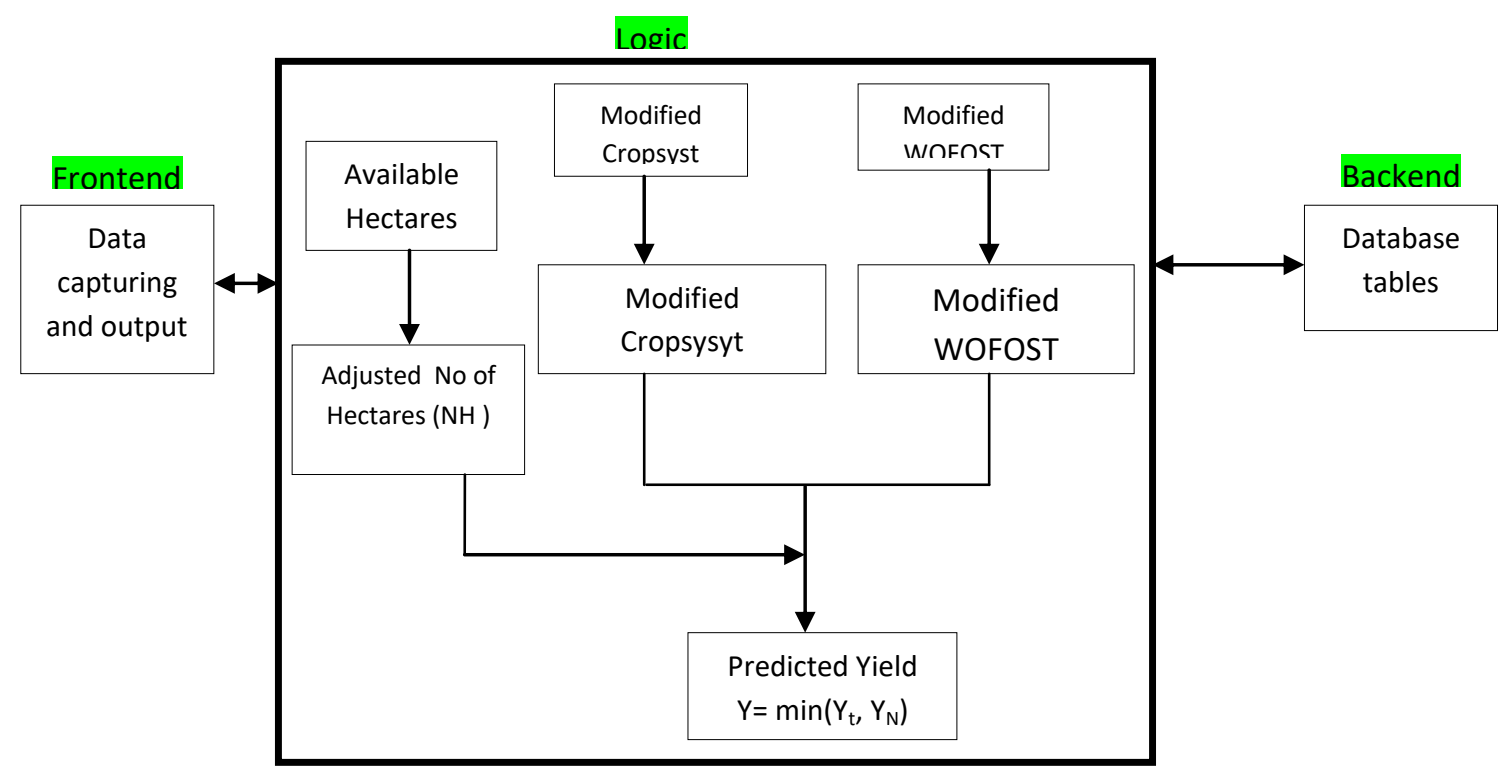


Figure 1.Architecture of the proposed model.

The front-end enables a two-way communication between the user and the system. This tier is responsible for receiving input and generation of reports. The input parameters of the proposed system are weather data, crisis data, soil fertility, crop parameters and location data. The sole report generated by the system is crop yield.
The logic tier is a collection of classes used in implementing the system. The various classes used as well their relationships are shown in figure 2.

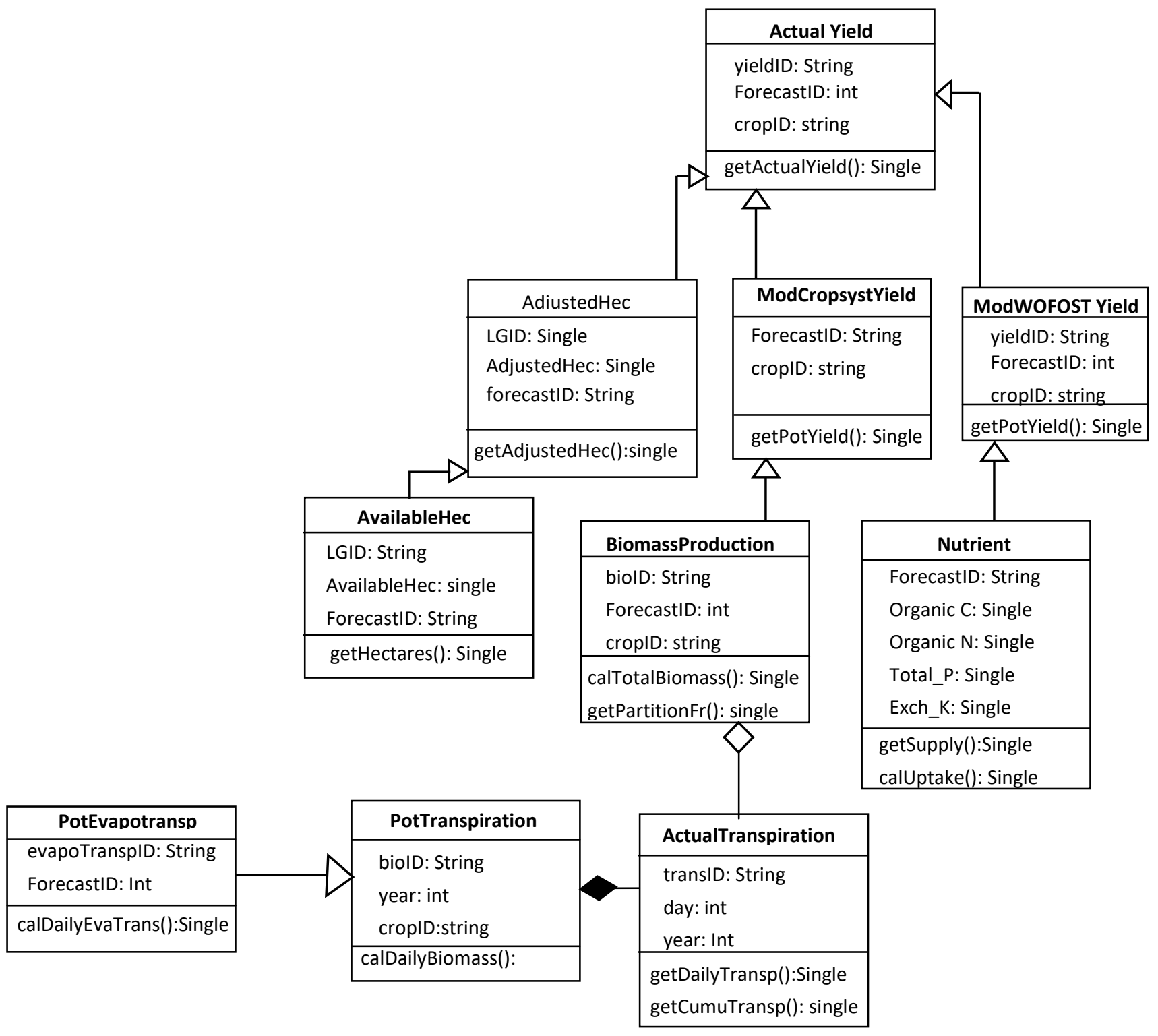

Fig 2: A class diagram of the proposed system 
In the work ten (10) classes were used. The most important of these classes is ActualYield which is responsible for the forecast of the actual crop yield. This class spawned three classes namely AdjustedHec, ModCrosystYield and ModWOFOSTYield. The three classes in turn spawned all other classes in the system. The three classes represent the three components of the logic tier. The first component handled crisis and the availability of hectares for farming activities and the classes in this group are AvailableHec and AdjustedHec. The second component handled the crop yield prediction using the modified Cropsyst model and the classes included ModCropsystYield, BiomassProduction, ActualTranspiration, Pottranspiration and PotEvatramp. The third component handled crop yield prediction using the modified WOFOST model. Classes in this group included ModWOFOSTYield and Nutrient.

The back-end of the proposed system is a database made up of nine (9) tables. The tables and the various relationships among them is as shown in the entity relationship diagram (ERD) in figure 3.

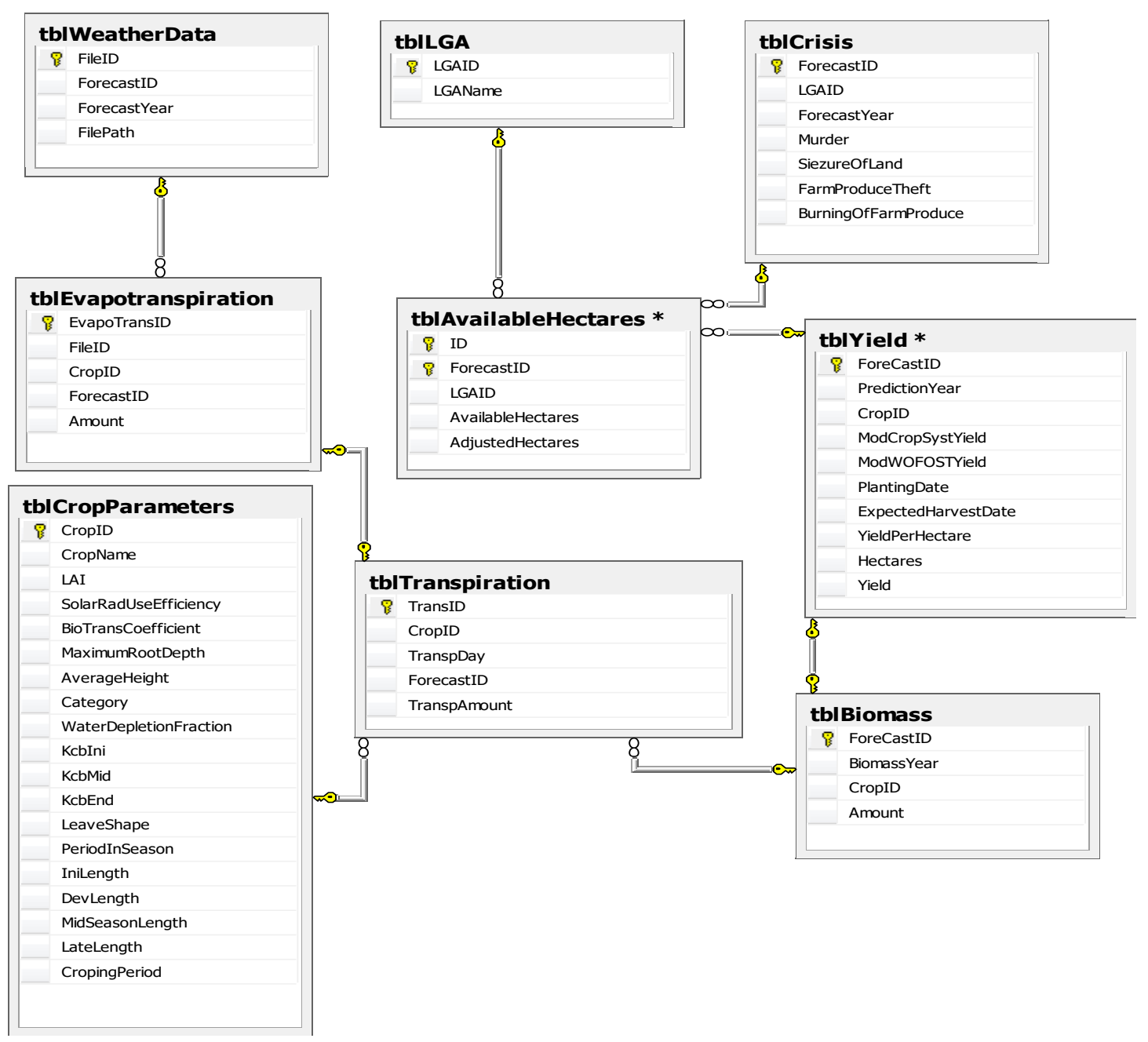

Figure 3.Entity relationship diagram of the proposed system 
In figure 3 , tblLGA stores the number of Local government areas (LGAs) in Benue state and available hectares of farmland, tblcrisis stores crisis data. Crisis data is represented in form of murder, land seizure and crop destruction with each carrying a designated value. E.g murder carries a value of 1. tblEvapotranspiration table stores the daily amount of water evaporated and

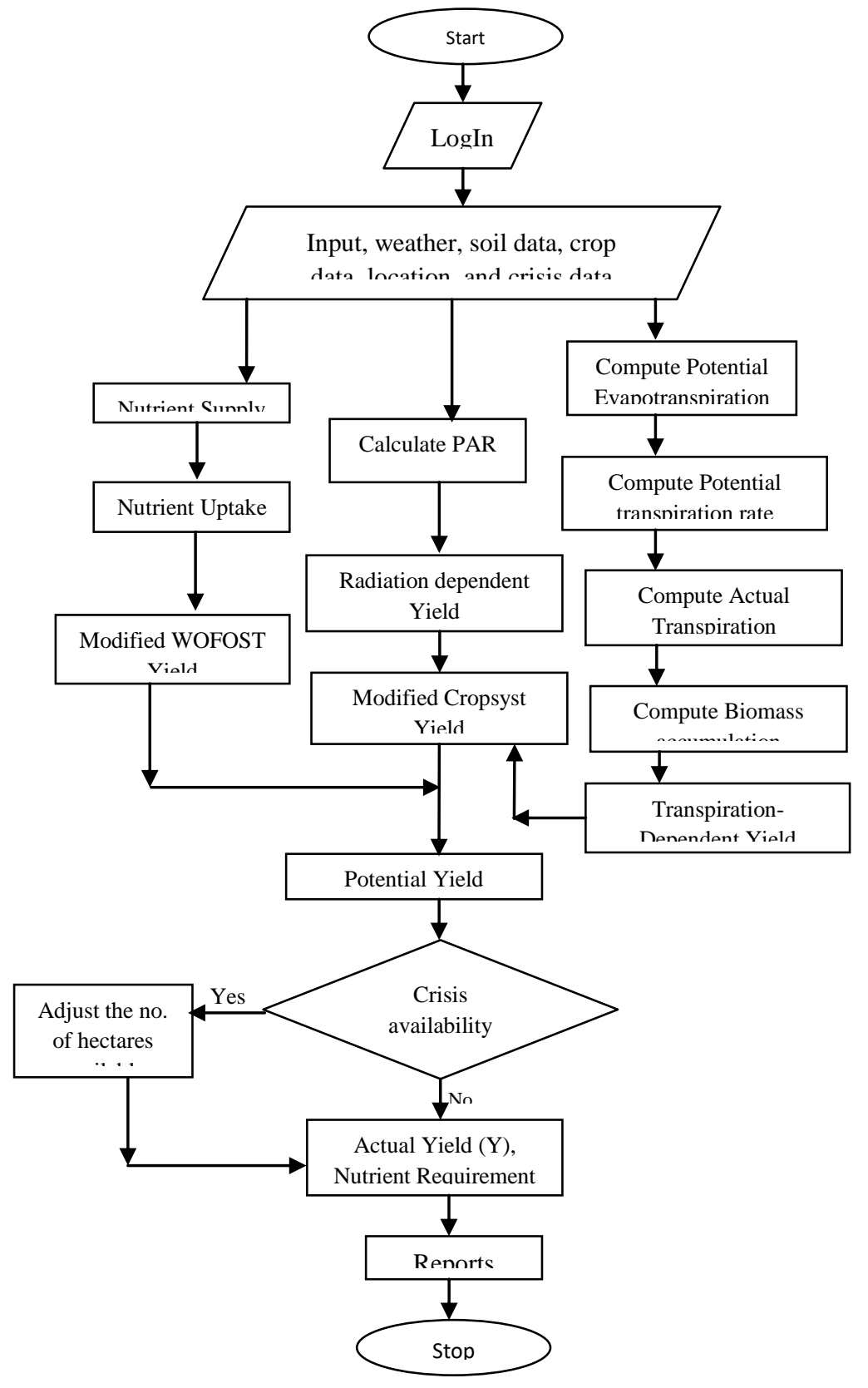

transpired into the atmosphere. tblcropparameters stores the crop specific properties like maximum root depth. tblAvailableHectares stores the number of hectares adjusted by crisis situation in an LGA. tblBiomass stores the daily accumulated crop biomass used in yield prediction, tblYield stores the predicted yield of a crop in a year.

Figure 4: A system chart for the proposed system 


\subsection{Detailed Design of the Model}

In figure 4 , the user starts the system and $\operatorname{logs}$ in. If successful, the user inputs weather, soil, crop, location and crisis data. Soil data is used to estimate the amount of $\mathrm{N}, \mathrm{P}$ and $\mathrm{K}$ in the soil and $\mathrm{N}, \mathrm{P}$ and $\mathrm{K}$ supply is used to estimate nutrient uptake of a crop.

Nutrient-limited crop yield is then estimated using the modified WOFOST module

Modified Cropsyst computes the yield $\left(\mathrm{Y}_{\mathrm{t}}\right)$ using weather data. The estimated yield from modified WOFOST and modified Cropsyst are used to compute Potential Yield. Availability of crisis adjusts the number of hectares available for production and actual crop yield $\left(\mathrm{Y}_{\mathrm{N}}\right)$ is computed. Reports are then generated and system stops.

\section{RESULTS AND DISCUSSION}

The model was simulated using Visual Basic 2010 and the back-end was implemented using Microsoft SQL Server 2008. Three (3) experiments were carried out involving years 2011, 2012 and 2013 and various crop yields generated. The three experiments utilized three different datasets for the three years. Each of the dataset comprise daily temperature, wind speed, sunshine and relative humidity from the planting date to expected harvest date for soybeans, maize, millet and sorghum. Each dataset also comprises latitude, rainfall and soil nutrient data. The results of the prediction were captured in table 1 .

Table 1: Predicted crop yield (in metric tons (MT)) for various crops and years.

\begin{tabular}{llll}
\hline Year & $\mathbf{2 0 1 1}$ & $\mathbf{2 0 1 2}$ & $\mathbf{2 0 1 3}$ \\
\hline Soybeans & & & 1.21 \\
Maize & 1.23 & 1.14 & 1.50 \\
Millet & 0.85 & 0.92 & 1.31 \\
Sorghum & 0.49 & 0.50 & - \\
\hline
\end{tabular}

Table 1 shows the predicted yield of soybeans, maize, millet and sorghum for year 2011, 2012 and 2013 respectively. Soybeans, maize, millet and sorghum had a predicted yield of 1.23MT, 0.85MT, 0.49MT and 1.20MT for year 2011, 1.14MT, 0.92MT, 0.50MT and 0.79MT for year 2012. In year 2013, soybeans, maize and millet had predicted yield of 1.21MT, 1.5MT and 1.31MT respectively.

\section{CONCLUSION}

In this work, WOFOST and Cropsyst models were hybridized and a crisis component added to the hybrid model and used in the prediction of yields for selected crops in Benue state, Nigeria, for three (3) years. The work used the two models to predict potential yield. Potential yield was modified using the crisis component to come up the predicted yield. The model was simulated using VB.net 2010. The results indicated high accuracy in yield prediction as related to crisis prone regions like Benue state in Nigeria, having extremely fertile lands and climate for agriculture purposes.

\subsection{Recommendation}

The work is recommended for use by stakeholders in the agricultural sector because of its accurate yield prediction. The work can also be used as a planning tool by stakeholders to 
determine the level of food availability in future especially if taken alongside population growth. For future research, we recommend the integration of several models and

\section{REFERENCES}

Anup K. P, Lim C, Ramesh P. S and Menas K 2006. Crop Yield estimation model for Iowa using remote sensing and surface parameters. International Journal of Applied Earth Observation and Geo information 8:26-33

Bornn, L. and Zidek, J. V. 2012. Efficient Stabilization of Crop Yield Prediction in the Canadian Praires. Agricultural and Forest Meteorology 152(2012) pp23232.

Bouman, B.A.M., Van Keulen, H., Van Laar H.H., and Rabbinge, R. 1996. The "School of de Wit" crop growth simulation models: A pedigree and historical overview. Agricultural Systems, 52(2-3), pp.171- 198.

Conor L and Mikahail A. S 2005. Assessing lead-time for predicting wheat growth using a crop crop simulation model. Agricultural and Forest Meteorology 135(2005) 302-313. Accessed on 11/10/2016 at www.elsevier.com/locate/agromaet.

Dahikar S.S. and Rode S.V. 2014. Agricultural Yield Prediction using Artificial Neural Networks Approach. International Journal of Innovative Research in Electrical, Electronics, Instrumentation and Control Engineering 2(1): 683-686

Galan, C., Gracia-Mozo, H., Vazques, L., Ruiz, L., Guardia D. and Domínguez-Vilches E. C. 2008. Modeling Olive Crop Yield in Andalusia, Spain. Agronomy Journal 100(1) pp98. Accessed on 11/10/2016 methodologies like neural-fuzzy, computer vision, GIS, satellite imaging, crisis modeling into a single model for more accurate crop yield prediction. http://www.uco.es/raa/publicaciones/andalucia/cordoba/m odelling_olive_andalusia_08.pdf.

Hoogenboom, G. and Gresham, D. D. 1993. Prediction of Spatial yield and water use in agriculture as a function of environmental conditions. Proceedings of 1993 Georgia Water Resources Conference held April 20 and 211993 at the University of Georgia.

Kefaya, Q. Evor, H. and Daciana, I. 2011. Recent research in Artificial Intelligence, Knowledge Engineering and Data Bases. Accessed on 13/11/2016 at http://www.wseas.us/elibrary/conferences/2011/Cambridg e/AIKED/AIKED-34.pdf

Kushwaha, A. K., Sweta B. 2015 Crop yield prediction using Agro Algorithm in Hadoop. IRACST-International Journal of Computer Science and Information Technology $\&$ Security (IJCSITS).

Nwajiuba C. 2012. Nigeria's Agriculture and Food Security Challenges. Accessed on 12/12/2015 at https://ng.boell.org/sites/default/files/uploads/2013/10/agr iculture_-_green_deal_nigeria_study.pdf

Savin, I. Y., Stathakis, D., Negre, T. and Isaev, V. A. 2007. Prediction of Crop Yields with the use of Neaural Networks. Russian Agricultural Sciences 33(6) pp361363. Allerton Press Inc

Sawasawa, H. L. 2003 Crop Yield Estimation: Integrating RS, GIS and Management Factors. A case study of Birkoor and Kortgiri mandals in Nizamabad district, India. Thesis submitted to the International Institute for Geo-information and Earth Observation Erischede, the Netherlands in partial fulfillment of the requirements for the degree of Master of Science in Geo-information science and Earth Obsrvation; Sustainable Agriculture. 
Stockle C. O. 1996. The CropSyst Model: A brief description. Biological Systems Engineering Dept. Washington State University. Accessed on 22/09/2014 at www.sciencedirect.com/science/article/pii/0308521X949 00062

Verma V, Piepho HP, Hartung K, Ogutu JO and Goyal A. 2015). Linear Mixed Modeling for mustard yield prediction in Haryana state (Iindia) Journal of Mathematics and Statistical Sciences 96-105.
Li A, Liang S, Wang A and Qin J 2007. Estimating crop yield from multi-temporal satellite data using multivariate regression and neural network techniques. Photogrammetric engineering \& Remote Sensing 73(10):

1149-1157

Wang, Q., Nuske, S., Bergerman, M. and Singh, S. 2012. Automated Crop Yield Estimation for Apple Orchards. In proc. International Symposium on Experimental robotics, Quebec City. 